\title{
Accurate estimation of choriocapillaris flow deficits beyond normal intercapillary spacing with swept source OCT angiography
}

\author{
Qinqin Zhang ${ }^{1}$, Yingying Shi ${ }^{2}$, Hao Zhou ${ }^{1}$, Giovanni Gregori ${ }^{2}$, Zhongdi Chu ${ }^{1}$, Fang Zheng ${ }^{2}$, Elie $\mathbf{H}$. \\ Motulsky $^{2}$, Luis de Sisternes ${ }^{3}$, Mary Durbin ${ }^{3}$, Philip J. Rosenfeld ${ }^{2}$, Ruikang K. Wang ${ }^{1,4}$ \\ ${ }^{1}$ Department of Bioengineering, University of Washington, Seattle, WA, USA; ${ }^{2}$ Department of Ophthalmology, Bascom Palmer Eye Institute, \\ University of Miami Miller School of Medicine, Miami, FL, USA; ${ }^{3}$ Advanced Development, Carl Zeiss Meditec, Inc., Dublin, CA, USA; \\ ${ }^{4}$ Department of Ophthalmology, University of Washington Eye Institute, Seattle, WA, USA
}

Correspondence to: Ruikang K. Wang, PhD. Department of Bioengineering, University of Washington, Seattle, WA, USA. Email: wangrk@uw.edu.

Background: To estimate choriocapillaris flow deficits beyond normal intercapillary distance with swept source optical coherence tomography angiography (SS-OCTA).

Methods: Subjects were enrolled and repeated SS-OCTA scans were performed using the $3 \mathrm{~mm} \times 3 \mathrm{~mm}$ scan pattern. Blood flow was identified using the complex optical microangiography (OMAG ${ }^{c}$ ) algorithm. The choriocapillaris (CC) was defined as a $20 \mu \mathrm{m}$ slab of the flow volume beneath the outer boundary of Bruch's membrane (BM) and was compensated with the corresponding structural image for flow deficits measurement. Flow deficits were segmented based on one mean standard deviation from a normal database. A histogram based thresholding method was developed to remove small flow deficits that were determined by examining intercapillary spacing within normal CC networks. A registration method based on affine and B-spline transformation was utilized for the CC angiogram averaging. Four repeated scans were averaged, and results were compared with and without removal of small flow deficits after averaging a different number of scans ( $\mathrm{N}=1$, group $1 ; \mathrm{N}=2$, group 2; $\mathrm{N}=3$, group 3 and $\mathrm{N}=4$, group 4).

Results: Seven normal subjects were enrolled. Intercapillary distance was found to be $24 \mu \mathrm{m}$ for the CC networks under OCTA, which was used as the threshold to exclude small flow deficits for CC quantification. After averaging, significant reduction in background noise and improvement in continuity of blood vessel networks were observed both on retinal and choriocapillaris angiograms. Flow deficit percentages of the choriocapillaris were significantly reduced with averaging (group 1 vs. group 2: $\mathrm{P}<0.0001$; group 2 vs. group 3: $\mathrm{P}<0.001$; group 3 vs. group 4: $\mathrm{P}<0.001)$. The flow deficit percentages were also significantly reduced after removing the small flow deficits $(\leq 24 \mu \mathrm{m}$ in diameter) in all groups $(\mathrm{P}<0.01)$. A statistically significant difference was found after removing small flow deficits $(\leq 24 \mu \mathrm{m}$ in diameter) between group 1 and group 2 $(\mathrm{P}<0.001)$, between group 2 and group $3(\mathrm{P}<0.05)$, and between group 3 and group $4(\mathrm{P}<0.05)$. However, the significance was decreased compared to that without small flow deficits removal.

Conclusions: A method was developed to improve the robust estimation of choriocapillaris flow deficits by removing the small flow deficits corresponding to normal intercapillary spacing. After the removal of small flow deficits, fewer repeats were required for image averaging to achieve comparable accuracy of flow deficit measurements with SS-OCTA.

Keywords: Intercapillary spacing; oxygen diffusion distance; optical coherence tomography angiography (OCTA); swept source OCTA; choriocapillaris, flow deficits

Submitted Aug 14, 2018. Accepted for publication Aug 21, 2018.

doi: 10.21037/qims.2018.08.10

View this article at: http://dx.doi.org/10.21037/qims.2018.08.10 


\section{Introduction}

As a non-invasive, high resolution and rapid imaging modality, optical coherence tomography angiography (OCTA) has become important for the diagnosis and management of retinal diseases (1-14). However, due to the strong scattering property of the retinal pigment epithelium (RPE) at the wavelength of $840 \mathrm{~nm}$, spectral domain OCTA (SD-OCTA) has difficulty in imaging the choroidal vasculature through an intact RPE $(15,16)$. In comparison, swept source OCTA (SS-OCTA) operating at a longer wavelength of $1,060 \mathrm{~nm}$ provides better imaging of the choroidal vasculature, including the choriocapillaris, due to less scattering by the RPE $(15,16)$. In addition, with a deeper penetration into the choroid and greater laser power due to the safer wavelength, SS-OCTA has a better signal to noise ratio when imaging the choriocapillaris.

SS-OCTA has been used to visualize the choriocapillaris $(11,14,17)$. The dense lobular pattern of the choriocapillaris in the central macula and the radially elongated lobules in the periphery were observed and the morphologies were compared with histopathologic specimens $(11,17)$. Quantitative assessments have focused on measurements of flow or signal deficits due to the difficulty of imaging directly the complex network of capillaries in the central macula. Spaide et al. calculated the distribution of choriocapillaris flow voids with SD-OCTA via a thresholding method in normal and diseased eyes and observed a power law distribution with parameters depending on age and disease (18). Using the same thresholding method, Sadda's group quantified vessel density (VD) and grey value (GV) of the choriocapillaris in normal subjects with SS-OCTA and found that both numbers were lower in the subfoveal field than that in the four parafoveal sections (19). It should be noted that the thresholding used in these methods was different for each individual image. Recently, we developed a novel method for choriocapillaris visualization and quantification in SSOCTA datasets (17). The shadowing effect caused by the $\mathrm{RPE} / \mathrm{BM}$ complex was compensated using the structural information from the same choriocapillaris slab. In addition, the method used histogram normalization and a single threshold determined from a normal database to characterize the flow voids in the en face choriocapillaris angiograms.

The accuracy of flow void measurements from the choriocapillaris can be further improved via image averaging. Even though 1,060 nm wavelength has deeper penetration, the relatively strong scattering of the RPE complex still prevents SS-OCTA from providing high quality images of the choriocapillaris. The limited lateral resolution of current imaging devices ( 15-20 um) and the relatively low density of the sampling protocol (with a spacing around $10 \mathrm{um}$ ) also reduce image quality in choriocapillaris imaging. In previous studies, averaging of multiple OCTA images largely improved the image quality by reducing noise and annealing discontinuous vessel segments $(20,21)$.

In this paper, we use the term flow deficits rather than flow or signal voids to describe the areas of low signal in the choriocapillaris slabs. It should be noted that the absence of OCTA signal may indicate absence of flow or flow that is below the detection sensitivity of the OCTA system. In addition, we explore the intercapillary distances (ICD), which have been measured to evaluate the uniformity of capillary spacing and used as an indicator of hypoxia in various organs and tissues (22-24). The normal capillary network is a uniform geometrical arrangement of blood vessels with various ICDs, and the distribution has been shown to be approximately log-normal (25). Since larger ICDs between microvessels may cause failure of oxygen diffusion or chronic hypoxia, it is important to identify flow deficits that are larger than the normal intercapillary spacing, and these flow deficits are expected to be more disease-related. It is known from histological studies (26) that the lobule sizes, capillary diameters, and ICDs vary from the central macula to the periphery. The dense capillary meshwork has an ICD ranging from 2 to $20 \mu \mathrm{m}$ in the posterior pole and a larger ICD of 200-300 $\mu \mathrm{m}$ towards the periphery. This phenomenon was also observed by SSOCTA imaging in vivo $(11,17)$. This suggests that focusing on the OCTA flow deficits larger than the ICD may represent an effective strategy.

In this paper, we propose to use the averaged ICD as a criterion to remove flow deficits identified by OCTA that are smaller than the normal intercapillary spacing. We demonstrate that this new strategy is able to achieve more accurate estimation of choriocapillaris flow deficits.

\section{Methods}

\section{Subjects}

All subjects were enrolled at the Bascom Palmer Eye Institute in a prospective OCT imaging study. All the subjects were under dilated exams to identify as normal 
controls. The Institutional Review Board of the University of Miami Miller School of Medicine approved the study, and informed consent was obtained from all subjects. The study was performed in accordance with the tenets of the Declaration of Helsinki and compliant with the Health Insurance Portability and Accountability Act of 1996.

\section{Image acquisition and scanning protocols}

The instrument used in this study was a SS-OCTA system (PLEX $^{\circledR}$ Elite 9000, Carl Zeiss Meditec, Inc., Dublin, CA, USA) as described in our previous study (17). Briefly, it is characterized by a central wavelength of $1,060 \mathrm{~nm}$ with a bandwidth of $100 \mathrm{~nm}$, resulting in an A-scan depth of $3.0 \mathrm{~mm}$ and a full-width at half maximal (FWHM) axial resolution of $\sim 5 \mu \mathrm{m}$ in tissue. The lateral resolution at the retinal surface has been estimated at $\sim 16 \mu \mathrm{m}$. FastTrac ${ }^{\mathrm{TM}}$ motion correction software was used while the images were acquired to minimize the subject motion artifacts. The $3 \mathrm{~mm} \times 3 \mathrm{~mm}$ scan pattern was used in this study. It contained 300 A-lines per B-scan and $300 \mathrm{~B}$-scan positions with 4 repeated scans at each fixed location. The complex optical microangiography (OMAG') algorithm was used to obtain the OCTA images, utilizing variations in both the intensity and phase information between sequential B-scans at the same location to generate the motion signal, which indicates blood flow $(1,27)$. A validated semi-automated segmentation algorithm was applied to identify relevant retinal layers (28), and manual corrections were carried out as necessary to ensure accurate segmentation. A $20 \mu \mathrm{m}$ thick slab beneath $\mathrm{BM}$ was defined as the choriocapillaris slab. A maximum projection was applied on the segmented flow volumes to generate the en face angiograms. Tailing artifacts were removed from the en face choriocapillaris images before further analysis (29). Images were excluded from the study if significant media opacity was present, if signal strength was less than seven as defined by manufacturer, or if there were severe motion artifacts. Four repeated volume scans were acquired from all the enrolled patients to test the influence of averaging on measuring flow deficits of the choriocapillaris.

\section{Compensation of signal attenuation}

The signal attenuation caused by the RPE/BM complex was compensated using a strategy previously reported (17). Briefly, the incident light is attenuated when passing through the RPE/BM complex due to its highly scattering property, therefore a shadow is cast beneath, especially in the presence of drusen. The shadowing effect under drusen is observed as decreased signals on both structural and flow en face choriocapillaris images when compared with non-drusen regions. Thus, the summation of structural signal from the same choriocapillaris slab was inverted and used to compensate for the attenuated flow signal in the choriocapillaris image.

\section{Choriocapillaris flow deficits measurement}

Flow deficits (known as flow voids in our previous study) were defined as a percentage of the area of the region with flow below a fixed threshold and the area of the entire scanned region. The threshold for flow deficits was determined by one standard deviation (SD) from a normal database (20 subjects at 20-39 years old). This threshold showed the best repeatability in measuring flow deficits both in normal and drusen cases (17).

\section{Removal of small choriocapillaris flow deficits}

Figure 1 shows an example of flow deficit measurements from a normal subject. To make the approach applicable to the protocols with different scanning pattern, all the input images were re-sampled from $300 \times 300$ to $512 \times 512$ pixels. The structure compensated choriocapillaris angiogram with tailing artifact removal is shown in Figure 1A. One SD threshold from the normal database was applied to isolate the flow deficits and obtain the flow deficit map, which is a binary image (Figure $1 B$ ) where the value of one indicates flow deficit. The percentage of flow deficits can be obtained by a summation of all the one values divided by the total scanned area. The overlay (Figure 1C) of the choriocapillaris angiogram and the flow deficit map demonstrates the interplay of capillaries and flow deficits. Different sizes of flow deficits were observed in the flow deficit map (Figure 1B), ranging from a few pixels to dozens of pixels. The histogram of the flow deficit sizes is plotted in Figure 1D, and the distribution shows two modes (30). Following a radial power spectrum analysis $(31,32)$, the valley value (i.e., the dip) shown in Figure $1 D$ of the bimodal histogram can be interpreted as the average intercapillary distance within the CC map. This value is then selected as a cut-off threshold to remove the small flow deficits, which would correspond to flow deficit areas that would not cause deficiency in oxygen diffusion.

We performed the above procedure on a normal database 

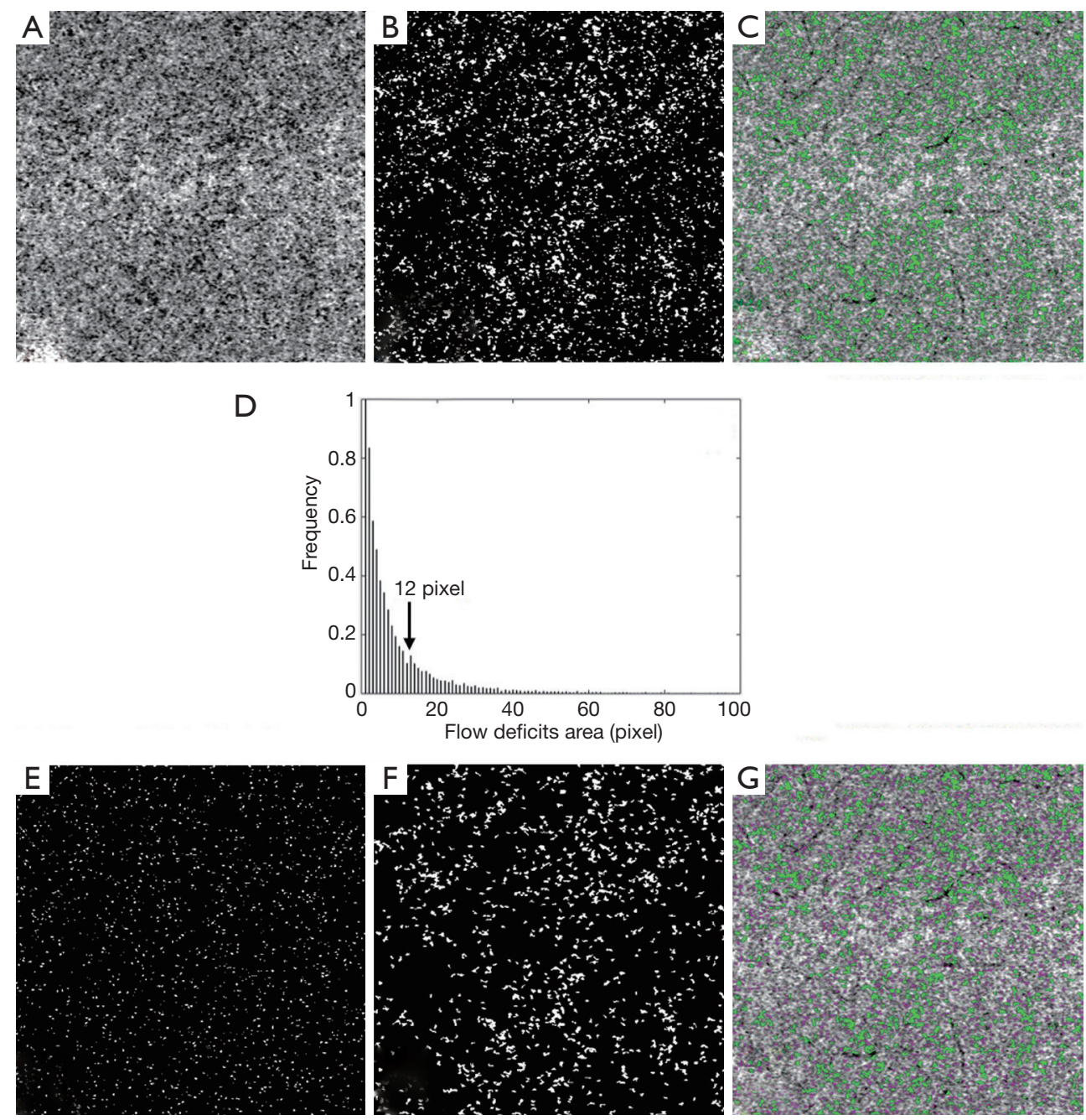

Figure 1 An example of the flow deficit measurement procedure using the thresholding method on an en face swept source optical coherence tomography angiography image of the choriocapillaris from a normal subject. (A) Structure compensated choriocapillaris en face angiogram with tailing artifact removed; (B) flow deficit map including all sizes of identified flow deficits; (C) overlaid choriocapillaris angiogram with flow deficits; (D) bi-modal histogram distribution showing the area sizes of flow deficits from which the average intercapillary distance is identified as 12 pixels from the valley value; (E) flow deficits smaller than intercapillary spacing; (F) the flow deficits larger than the intercapillary spacing, which are considered as true flow deficits; $(\mathrm{G})$ the overlaid choriocapillaris angiogram with small flow deficits in purple and true flow deficits in green.

with twenty young subjects with an age ranging from 20 to 39 years old. Twenty valley values were collected and averaged as a general threshold. The mean valley value was found to be 12 pixels in area size. By considering the flow deficit as a circle in the calculation, the diameter can be obtained which is $\sim 24 \mu \mathrm{m}$, representing the averaged ICD that is considered to be within normal intercapillary spacing and within normal oxygen diffusion capability. Figure $1 E$ shows the flow deficits, each with an area with an equivalent diameter smaller than $24 \mu \mathrm{m}$. These small flow deficits are removed from the flow deficit map. As a result, the remaining flow deficits (Figure $1 F$ ) should provide a depiction of flow deficits that represent oxygen diffusion limited areas. Figure $1 G$ shows an overlay image that includes the choriocapillaris angiogram with removed small flow deficits in purple and the remaining large flow deficits in green. 


\section{Image averaging}

To validate our method, four repeated scans were collected on each subject. For each imaged eye, angiograms were averaged with 1, 2, 3 and 4 scans, classified as group 1, group 2, group 3 and group 4, respectively. A registration method based on affine and B-spline transformation was utilized to align the repeated scans (33), using the superficial retinal layers as the reference to obtain the transformation parameters. Then, the registered images of each layer (retinal and compensated choriocapillaris) were merged into one image by averaging the images in each group.

Flow deficits with and without the small flow deficits removal were calculated on each group (single image and averaged images). Comparisons were performed between groups with and without the removal of small flow deficits.

\section{Statistical analysis}

A paired sample $t$-test was used to compare the flow deficits percentages between groups, as well as in each group with and without the small flow deficits removal. All statistical analyses were performed using Matlab ${ }^{\mathrm{TM}}$. $\mathrm{P}$ values below 0.05 were considered as statistically significant.

\section{Results}

Images from seven eyes of seven normal subjects were captured for this study. For each eye, four repeated scans were collected and averaged to test the influence of averaging on the flow deficit measurements with and without removal of the small flow deficits.

\section{Choriocapillaris imaging with averaging}

Figure 2 shows an example of retinal and choriocapillaris angiograms in four groups, including a single image and images averaged 2, 3 and 4 times. Generally, the image quality improves progressively with the number of averaged scans (Figure 2B,C,D,E,F,H) and so does the signal to noise ratio. When averaging 4 scans the background noise is significantly reduced and the visualization of the blood vessel networks is improved, showing greater continuity compared with the single image (Figure $2 A, E$ ).

\section{Flow deficit measurements of the choriocapillaris with and without averaging and removing small flow deficits}

The averaged choriocapillaris angiograms (Figure 2F,G,H) show a more continuous meshwork compared to the single image. We calculated the flow deficits on each group using the method described above. To avoid possible offsets at the edges of the registered images caused by variability in fixation, flow deficit percentages were calculated within a circle of diameter $2.5 \mathrm{~mm}$ (Figure 2I, $, \mathcal{H}, K, L$ ) centered on the fovea.

Generally, without removing the flow deficits that are with an area of equivalent diameter smaller than $24 \mathrm{um}$, flow deficits percentage decreased with increased averaging (Figure $2 I, \mathcal{F}, K, L)$. Increased averaging results in fewer flow deficits, and the value tends to become stable after averaging more images (Figure 3). Statistically significant differences in flow deficit measurements were found between groups (group 1 vs. group 2: $\mathrm{P}<0.0001$; group 2 vs. group 3: $\mathrm{P}<0.001$; group 3 vs. group $4: \mathrm{P}<0.001)$. After removing the small deficits ( $\leq 24 \mu \mathrm{m}$ in diameter), the flow deficit percentages decreased with increased averaging in all groups. A statistically significant difference was found between group 1 and group $2(\mathrm{P}<0.001)$, between group 2 and group $3(\mathrm{P}<0.05)$, and between group 3 and group 4 $(\mathrm{P}<0.05)$ after removing the small flow deficits $(\leq 24 \mu \mathrm{m}$ in diameter). However, the significant difference was decreased compared to without small flow deficits removal.

\section{Discussion}

Flow deficits were defined as regions of non-perfusion or low perfusion, where the flow was below the sensitivity limit of the current OCTA technology. They appeared as dark or weak signals on the OCTA angiograms. Even though SS-OCTA has deeper penetration and weaker light scattering at the RPE, which should reduce potential errors in flow deficit measurements when compared with SDOCTA, this definition of flow deficits can complicate the identification of these regions by several aspects: e.g., (I) noise characteristics of OCTA imaging can cause small flow voids to be variable, and (II) all normal eyes would contain small flow deficits consistent with the normal intercapillary distances. Our novel strategy for choriocapillaris visualization and quantification demonstrated here attempts to identify and discard a subset of flow voids that appear not to be physiologically relevant and are more likely to correspond to noise.

We have proposed a simple histogram based method to identify the averaged intercapillary spacing of the scanned OCTA en face choriocapillaris angiograms. This method provided an average of $24 \mu \mathrm{m}$ in equivalent spacing 

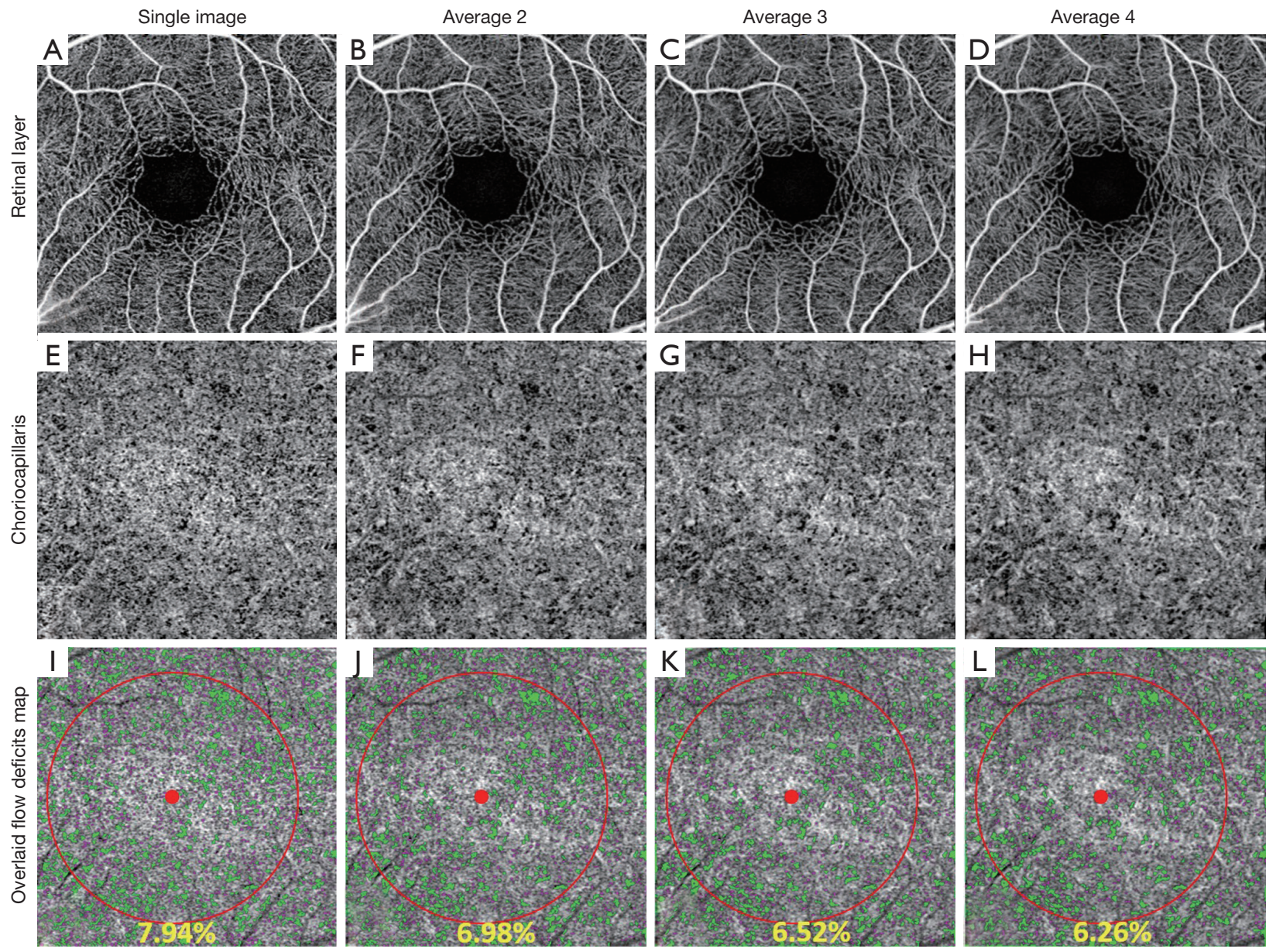

Figure 2 Example of retinal and choriocapillaris angiograms from a single image and images averaged 2, 3 and 4 times. (A,B,C,D) Retinal angiograms without averaging and averaged 2, 3 and 4 times; (E,F,G,H) choriocapillaris angiograms without averaging and averaged 2, 3 and 4 times; (I,J,K,L) flow deficit measurements based on a single image and images averaged 2, 3 and 4 times. Purple indicates the small flow deficits that are consistent with normal ICD; green indicates the true flow deficits identified through the procedure, and the yellow percentage indicates the percent of true flow deficits within the red circle. ICD, intercapillary distances.

diameter, as determined from a normal database with ages ranging from 20 to 39 years old.

According to a histology study (29), the intercapillary distance varies in different spatial locations within the fundus. An intercapillary distance ranging from 2 to $20 \mu \mathrm{m}$ has been reported in the posterior pole where there is a dense capillary meshwork; however, the multiple steps necessary for the preparation of a histologic sample preparation, may affect the true distance between capillaries. Adaptive optics (AO) OCTA with a high lateral resolution about $4.7 \mu \mathrm{m}$ has been adopted for choriocapillaris imaging by Miller's group (32). Although the field of view was limited to $4 \times 2$ degrees, the dense meshwork around central macula was clearly observed with a regular lobular pattern. The averaged spacing between choriocapillaris lumens was calculated based on the en face angiogram, at about $39 \mu \mathrm{m}$. In another study using OCTA (14), a fast imaging device with a $1.7 \mathrm{MHz}$ A-scan rate was utilized to visualize the choriocapillaris. With this imaging speed, it was possible to have a faster B-scan rate and denser scans with a step size to $4 \mu \mathrm{m}$ between adjacent $\mathrm{A}$-scans, allowing the observation of the lobular patterns under central macula. A spatial frequency of 25 cycles $/ \mathrm{mm}$ of the choriocapillaris peak using the radial power spectrum analysis method was reported, resulting in a capillary spacing at about $40 \mu \mathrm{m}$. However, current commercial OCTA devices have limited 


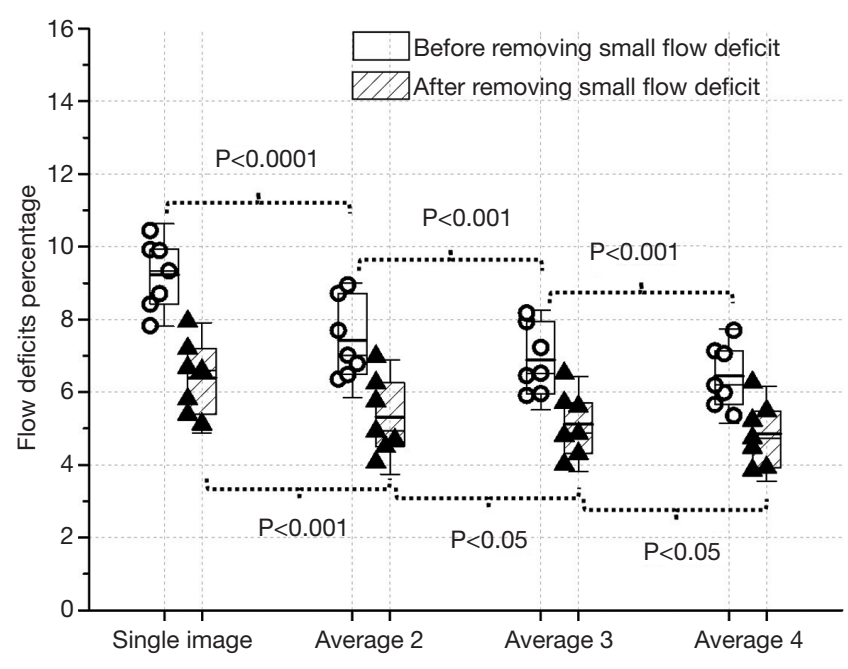

Figure 3 Box-plot of flow deficits measurement in all four groups.

lateral resolution $(\sim 15-20 \mu \mathrm{m})$ and lower density sampling with a step size of about $10 \mu \mathrm{m}$. Specifically, in our study, the device has a lateral resolution of about $16 \mu \mathrm{m}$ in retinal tissue and sampling space at about $10 \mu \mathrm{m}$. The relatively low lateral resolution compared to AO-OCTA (16 vs. $4.7 \mu \mathrm{m})$ and large sampling distance $(10 \mathrm{vs} .4 \mu \mathrm{m})$ are likely to generate choriocapillaris flow images with a dilated capillary meshwork (34). Consequently, the intercapillary spacing would likely be reduced. In our study, a bimodal distribution of the flow deficits was clearly observed in all twenty normal subjects. The averaged valley value from the normal database that resulted in a $24 \mu \mathrm{m}$ spacing in diameter can be well explained by considering the convolution between OCT probe beam spot size and the true intercapillary spacing.

Repeated scans were acquired on each subject to investigate the influence of averaging (4 groups) on flow deficit measurements. More averaging yielded a lower flow deficit percentage that appeared to become stable after averaging more images. This statistically significant decrease in flow deficit percentage demonstrated the advantages of averaging, which reduced background noise and improved the continuity of blood vessel networks. The flow deficit percentages were significantly reduced $(\mathrm{P}<0.01)$ in all groups after removing the small flow deficits $(24 \mu \mathrm{m}$ in diameter). This observation indicates that there may be a fixed bias between the results that are with and without small flow deficits removal. The fixed bias could come from the normal intercapillary spacing and could also be introduced by the system noises. The statistical differences were decreased after the small flow deficits $(\leq 24 \mu \mathrm{m}$ in diameter) removal, indicating the removal strategy may provide more robust flow deficits measurements with less averaging.

Limitations of this study include a limited sample size of 7 normal subjects with repeated scans. Another limitation is that only 4 repeated scans were acquired for each subject. It could be interesting to investigate results when more than 4 images are averaged. In addition, eyes with choriocapillaris pathologies should also be enrolled in future studies to investigate how the averaging affects the flow deficit measurements and how robust the method of small flow deficits removal can be in different diseases. For instance, in eyes with age-related macular degeneration that usually have large flow deficits, less averaging or no averaging may be necessary to achieve comparable accuracy for flow deficit measurements.

In conclusion, we have proposed a novel method to remove small flow deficits that correspond to a smaller than expected intercapillary spacing, reducing the effect of compounding factors, e.g., system noise and normal intercapillary distance, on the choriocapillaris flow deficit measurements. With this method, fewer repeats for image averaging were required to achieve comparable quantification on the flow deficits based on SS-OCTA imaging. This method should provide more accurate measurements of flow deficits that may help facilitate early diagnosis, monitoring disease progression, response to treatment, and prognosis.

\section{Acknowledgements}

Funding: Research supported by grants from Carl Zeiss Meditec, Inc. (Dublin, CA), the National Eye Institute (R01EY024158, R01EY028753), an unrestricted grant from the Research to Prevent Blindness, Inc., New York, NY, and the National Eye Institute Center Core Grant (P30EY014801) to the Department of Ophthalmology, University of Miami Miller School of Medicine.

\section{Footnote}

Conflicts of Interest: Drs. Gregori, Rosenfeld and Wang received research support from Carl Zeiss Meditec, Inc. Dr. Gregori and the University of Miami co-own a patent that is licensed to Carl Zeiss Meditec, Inc. Dr. Rosenfeld also received additional research support from Genentech, and Tyrogenex. He is a consultant for Achillion 
Pharmaceuticals, Acucela, Boehringer-Ingelheim, Carl Zeiss Meditec, Cell Cure Neurosciences, Chengdu Kanghong Biotech, Ocunexus Therapeutics, Genentech, Healios K.K, Hemera Biosciences, F. Hoffmann-La Roche Ltd., Isarna Pharmaceuticals, Lin Bioscience, MacRegen Inc, NGM Biopharmaceuticals, Ocunexus, Ocudyne, Tyrogenex, and Unity Biotechnology. Dr. Rosenfeld as has equity interest in Apellis, Digisight, and Ocudyne. Dr. Wang and the Oregon Health \& Science University co-own a patent that was licensed to Carl Zeiss Meditec, Inc. and Kowa Inc. Dr. Wang received an innovative research award from Research to Prevent Blindness. He is a consultant to Carl Zeiss Meditec, Insight Photonic Solutions. Drs. Durbin, and de Sisternes are employed by Carl Zeiss Meditec, Inc. Other authors have no conflicts of interest to declare.

Ethical Statement: The Institutional Review Board of the University of Miami Miller School of Medicine approved the study, and informed consent was obtained from all subjects.

\section{References}

1. Wang RK, An L, Francis P, Wilson DJ. Depth-resolved imaging of capillary networks in retina and choroid using ultrahigh sensitive optical microangiography. Opt Lett 2010;35:1467-9.

2. Chen CL, Wang RK. Optical Coherence Tomography Based Angiography. Biomed Opt Express 2017;8:1056-82.

3. Kashani AH, Chen CL, Gahm JK, Zheng F, Richter GM, Rosenfeld PJ, Shi Y, Wang RK. Optical coherence tomography angiography: A comprehensive review of current methods and clinical applications. Prog Retin Eye Res 2017;60:66-100.

4. Takase N, Nozaki M, Kato A, Ozeki H, Yoshida M, Ogura Y. Enlargement of foveal avascular zone in diabetic eyes evaluated by en face optical coherence tomography angiography. Retina 2015;35:2377-83.

5. Lin AD, Lee AY, Zhang Q, Rezaei KA, Kinyoun J, Wang RK, Lee CS. Association between OCT-based microangiography perfusion indices and diabetic retinopathy severity. Br J Ophthalmol 2017;101:960-4.

6. Zheng F, Zhang Q, Motulsky EH, de Oliveira Dias JR, Chen CL, Chu Z, Miller AR, Feuer W, Gregori G, Kubach S, Durbin MK, Wang RK, Rosenfeld PJ. Comparison of Neovascular Lesion Area Measurements From Different Swept-Source OCT Angiographic Scan Patterns in Age-Related Macular Degeneration. Invest
Ophthalmol Vis Sci 2017;58:5098-104.

7. Rezaei KA, Zhang Q, Chen CL, Chao J, Wang RK. Retinal and choroidal vascular features in patients with retinitis pigmentosa imaged by OCT based microangiography. Graefes Arch Clin Exp Ophthalmol 2017;255:1287-95.

8. Spaide RF. Optical Coherence Tomography Angiography Signs of Vascular Abnormalization With Antiangiogenic Therapy for Choroidal Neovascularization. Am J Ophthal 2015;160:6-16.

9. Zhang Q, Wang RK, Chen CL, Legarreta AD, Durbin MK, An L, Sharma U, Stetson PF, Legarreta JE, Roisman L, Gregori G, Rosenfeld PJ. Swept source optical coherence tomography angiography of neovascular macular telangiectasia Type 2. Retina 2015;35:2285-99.

10. Roisman L, Zhang Q, Wang RK, Gregori G, Zhang A, Chen CL, Durbin MK, An L, Stetson PF, Robbins G, Miller A, Zheng F, Rosenfeld PJ. Optical Coherence Tomography Angiography of Asymptomatic Neovascularization in Intermediate Age-Related Macular Degeneration. Ophthalmology 2016;123:1309-19.

11. Choi W, Mohler KJ, Potsaid B, Lu CD, Liu JJ, Jayaraman V, Cable AE, Duker JS, Huber R, Fujimoto JG.

Choriocapillaris and choroidal microvasculature imaging with ultrahigh speed OCT angiography. PLoS One 2013;8:e81499.

12. Nesper PL, Soetikno BT, Fawzi AA. Choriocapillaris Nonperfusion is Associated With Poor Visual Acuity in Eyes With Reticular Pseudodrusen. Am J Ophthalmol 2017;174:42-55.

13. Choi W, Moult EM, Waheed NK, Adhi M, Lee B, Lu CD, de Carlo TE, Jayaraman V, Rosenfeld PJ, Duker JS, Fujimoto JG. Ultrahigh-Speed, Swept-Source Optical Coherence Tomography Angiography in Nonexudative Age-Related Macular Degeneration with Geographic Atrophy. Ophthalmology 2015;122:2532-44.

14. Gorczynska I, Migacz JV, Jonnal R, Zawadzka RJ, Werner JS. Imaging of the human choroid with a $1.7 \mathrm{MHz}$ A-scan rate FDML swept source OCT system. In: Manns F, Söderberg PG, Ho A, editors. Ophthalmic Technologies XXVII 2017;10045:1004510.

15. Zhang Q, Chen CL, Chu Z, Zheng F, Miller A, Roisman L, Rafael de Oliveira Dias J, Yehoshua Z, Schaal KB, Feuer W, Gregori G, Kubach S, An L, Stetson PF, Durbin MK, Rosenfeld PJ, Wang RK. Automated Quantitation of Choroidal Neovascularization: A Comparison Study Between Spectral-Domain and Swept-Source OCT Angiograms. Invest Ophthalmol Vis Sci 2017;58:1506-13. 
16. Miller AR, Roisman L, Zhang Q, Zheng F, Rafael de Oliveira Dias J, Yehoshua Z, Schaal KB, Feuer W, Gregori G, Chu Z, Chen CL, Kubach S, An L, Stetson PF, Durbin MK, Wang RK, Rosenfeld PJ. Comparison Between Spectral-Domain and Swept-Source Optical Coherence Tomography Angiographic Imaging of Choroidal Neovascularization. Invest Ophthalmol Vis Sci 2017;58:1499-505.

17. Zhang Q, Zheng F, Motulsky EH, Gregori G, Chu Z, Chen CL, Li C, de Sisternes L, Durbin M, Rosenfeld PJ, Wang RK. A Novel Strategy for Quantifying Choriocapillaris Flow Voids Using Swept-Source OCT Angiography. Invest Ophthalmol Vis Sci 2018;59:203-11.

18. Spaide RF. Choriocapillaris Flow Features Follow a Power Law Distribution: Implications for Characterization and Mechanisms of Disease Progression. Am J Ophthalmol 2016;170:58-67.

19. Al-Sheikh M, Falavarjani KG, Pfau M, Uji A, Le PP, Sadda SR. Quantitative Features of the Choriocapillaris in Healthy Individuals Using Swept-Source Optical Coherence Tomography Angiography. Ophthalmic Surg Lasers Imaging Retina 2017;48:623-31.

20. Uji A, Balasubramanian S, Lei J, Baghdasaryan E, AlSheikh M, Sadda SR. Impact of Multiple En Face Image Averaging on Quantitative Assessment from Optical Coherence Tomography Angiography Images. Ophthalmology 2017;124:944-52.

21. Mo S, Phillips E, Krawitz BD, Garg R, Salim S, Geyman LS, Efstathiadis E, Carroll J, Rosen RB, Chui TY. Visualization of Radial Peripapillary Capillaries Using Optical Coherence Tomography Angiography: The Effect of Image Averaging. PLoS One 2017;12:e0169385.

22. Awwad HK, el Naggar M, Mocktar N, Barsoum M. Intercapillary distance measurement as an indicator of hypoxia in carcinoma of the cervix uteri. Int J Radiat Oncol Biol Phys 1986;12:1329-33.

23. Salathé EP, Wang TC, Gross JF. Mathematical analysis of oxygen transport to tissue. Math Biosci 1980;51:89-115.

24. Tan PE, Balaratnasingam C, Xu J, Mammo Z, Han SX, Mackenzie P, Kirker AW, Albiani D, Merkur AB, Sarunic MV, Yu DY. Quantitative Comparison of Retinal Capillary Images Derived By Speckle Variance Optical Coherence Tomography With Histology. Invest Ophthalmol Vis Sci 2015;56:3989-96.

25. Turek Z, Rakuŝan K. Lognormal distribution of intercapillary distance in normal and hypertrophic rat heart as estimated by the method of concentric circles: its effect on tissue oxygenation. Pflugers Arch 1981;391:17-21.

26. Olver JM. Functional Anatomy of the Choriodal Circulation: Methyl Methacrylate Casting of Human Chorid. Eye 1990;4:262-72.

27. An L, Subhush HM, Wilson DJ, Wang RK. Highresolution wide-field imaging of retinal and choroidal blood perfusion with optical microangiography. J Biomed Opt 2010;15:026011.

28. Yin X, Chao JR, Wang RK. User-guided segmentation for volumetric retinal optical coherence tomography images. J Biomed Opt 2014;19:086020.

29. Zhang A, Zhang Q, Wang RK. Minimizing projection artifacts for accurate presentation of choroidal neovascularization in OCT micro-angiography. Biomed Opt Express 2015; 6:4130-43.

30. Raju PDR, Neelima G. Image segmentation by using histogram thresholding, IJCSET 2012;2:776-9.

31. Prabhakar S, Naidu MP. Power spectrum and its applications. In: Naidu PS, Mathew MP. Analysis of Geophysical Potential Fields 1998;5:75-143.

32. Kurokawa K, Liu Z, Miller DT. Adaptive optics optical coherence tomography angiography for morphometric analysis of choriocapillaris [Invited]. Biomed Opt Express 2017;8:1803-22.

33. Wei DW, Deegan AJ, Wang RK. Automatic motion correction for in vivo human skin optical coherence tomography angiography through combined rigid and nonrigid registration. J Biomed Opt 2017;22:66013.

34. Salas M, Augustin M, Ginner L, Kumar A, Baumann B, Leitgeb R, Drexler W, Prager S, Hafner J, SchmidtErfurth U, Pircher M. Visualization of micro-capillaries using optical coherence tomography angiography with and without adaptive optics. Biomed Opt Express 2016;8:207-22.

Cite this article as: Zhang Q, Shi Y, Zhou H, Gregori G, Chu Z, Zheng F, Motulsky EH, de Sisternes L, Durbin M, Rosenfeld PJ, Wang RK. Accurate estimation of choriocapillaris flow deficits beyond normal intercapillary spacing with swept source OCT angiography. Quant Imaging Med Surg 2018;8(7):658-666. doi: 10.21037/qims.2018.08.10 